\title{
The Influence of Vertical Wind Shear on Moist Thermals
}

\author{
JOHN M. PETERS \\ Department of Meteorology, Naval Postgraduate School, Monterey, California \\ WALTER HANNAH \\ Lawrence Livermore National Laboratory, Livermore, California
}

HugH MORRISON

Mesoscale and Microscale Meteorology Laboratory, National Center for Atmospheric Research, Boulder, Colorado

(Manuscript received 1 October 2018, in final form 12 March 2019)

\begin{abstract}
Although it is well established that vertical wind shear helps to organize and maintain convective systems, there is a longstanding colloquial notion that it inhibits the development of deep convection. To investigate this idea, the vertical momentum budgets of sheared and unsheared moist thermals were compared in idealized cloud model simulations. Consistent with the idea of vertical wind shear inhibiting convective development, convection generally deepened at a slower rate in sheared simulations than in unsheared simulations, and the termination heights of thermals in sheared runs were correspondingly lower. These differences in deepening rates resulted from weaker vertical acceleration of thermals in the sheared compared to the unsheared runs. Downward-oriented dynamic pressure acceleration was enhanced by vertical wind shear, which was the primary reason for relatively weak upward acceleration of sheared thermals. This result contrasts with previous ideas that entrainment or buoyant perturbation pressure accelerations are the primary factors inhibiting the growth of sheared convection. A composite thermal analysis indicates that enhancement of dynamic pressure acceleration in the sheared runs is caused by asymmetric aerodynamic lift forces associated with shear-driven cross flow perpendicular to the direction of the thermals' ascent. These results provide a plausible explanation for why convection is slower to deepen in sheared environments and why slanted convection tends to be weaker than upright convection in squall lines.
\end{abstract}

\section{Introduction}

The interaction of convection with vertical wind shear has been a popular research topic for decades. This interaction is perhaps best encapsulated by a statement from Takeda (1966): "vertical wind shear does not intensify the convective system itself, but organizes the release of convective-instability energy by maintaining the convective system and causing it to propagate." Indeed, early simulations by cloud-resolving models (CRMs) in the late 1970s and early 1980s showed that shear regulates convective organization, promoting the existence of long-lived multicellular clusters, squall lines, and supercell thunderstorms (e.g., Klemp and Wilhelmson 1978; Weisman and Klemp 1982, hereafter WK82). The pressure perturbations resulting from

Corresponding author: John M. Peters, jmpeters@nps.edu cumulus interactions with shear allow them to propagate in directions that are substantially different than the mean tropospheric flow (e.g., Klemp and Wilhelmson 1978; Rotunno and Klemp 1982; Weisman and Klemp 1984; Weisman and Rotunno 2000; Davies-Jones 2002). Low-level streamwise vorticity associated with low-level shear is the source of rotation in supercell thunderstorms (Davies-Jones 1984, 2002). Dynamically driven lift along outflow boundaries in sheared environments plays a key role in initiating and maintaining convective updrafts (e.g., Hane 1973; Thorpe et al. 1982; Rotunno et al. 1988; Weisman 1992, 1993; Moncrieff and Liu 1999; Bryan et al. 2006; Bryan and Rotunno 2014). In steady long-lived supercell updrafts, strong low-level stormrelative flow associated with vertical wind shear makes updrafts wide (Warren et al. 2017; Trapp et al. 2017) and thus reduces their susceptibility to entrainment-driven dilution (Hannah 2017; Peters et al. 2019, manuscript 
submitted to J. Atmos. Sci.). Shear may also affect storm morphology by modulating the positioning of precipitation and rain-cooled downdrafts relative to a cloud's updraft (e.g., Rasmussen and Straka 1998). The theories developed by the aforementioned studies are well supported by observational analyses, and have been used to develop successful forecasting tools (e.g., Brooks et al. 1993; Bunkers et al. 2000; Thompson et al. 2003; Coniglio et al. 2007; Thompson et al. 2007; Smith et al. 2012; Thompson et al. 2012).

The literature summarized in the previous paragraph is primarily focused on the mature phase of deep convection. Comparatively little attention has been paid to the role of shear on developing convection (e.g., cumulus congestus clouds), which has applications to forecasts of convection initiation, convective trigger functions in cumulus parameterizations (CPs), and the convective adjustment time scale in cumulus parameterizations with prognostic closure (e.g., Pan and Randall 1998). Based on a remark in Markowski and Richardson (2010, p. 193) and anecdotal remarks from weather forecasters and storm chasers, the colloquial notion is that, while shear is beneficial for the longevity of organized deep convection, it substantially inhibits the initial development of convection. Given the idea that mechanical mixing between a cloud and its surrounding environment drives cloud core dilution, this colloquial notion makes intuitive sense. Furthermore, Parker (2010) and Peters (2016) argued that slanted updrafts should be weaker than their upright counterparts-all else being equal-because of the larger downward-oriented buoyancy pressure perturbation acceleration in the former. Perhaps updrafts that develop in shear are more slanted than their unsheared counterparts, which reduces their rate of maturing into deep convection? The general lack of well-established support for the aforementioned arguments motivates further investigation of the role of shear in the development of deep convection.

To set the stage for our study, we review recent advances in understanding of the fundamental structure of deep convection. Recent large-eddy simulation studies have almost unanimously supported the view of convection as a grouping of quasi-spherical buoyant bubbles with toroidal circulations, referred to as "moist thermals" (or simply thermals; e.g., Zhao and Austin 2005; Sherwood et al. 2013; Romps and Charn 2015; Hernandez-Deckers and Sherwood 2016, hereafter DS). An essential element of convective initiation is the so-called preconditioning process, whereby initial ill-fated moist thermals add water vapor to the ambient environment as they decay and facilitate the survival of subsequent thermals (e.g., Damiani et al. 2008; Moser and Lasher-Trapp 2017). Because of the importance of moist thermals in developing convection, a natural starting point to our investigation is the analysis of the influence of shear on moist thermal dynamics. Modeling and laboratory studies of ring vortices that include environmental flow that travels in a perpendicular direction to the ring vortex (cross flow) show complex behavior, including both upshear and downshear tilting of the vortices as a response to different cross-flow magnitudes and vortex initialization methods (e.g., Fric and Roshko 1994; Chang and Vakili 1995; Sau and Mahesh 2008). Airborne observational studies of cumulus congestus clouds show that a thermal's internal circulation sometimes tilts upstream in sheared flow because of opposing upward and downward aerodynamic lift on the upshear and downshear flanks of the toroidal circulation, respectively (e.g., Chang and Vakili 1995; Damiani and Vali 2007). In a simulation of sheared shallow cumulus clouds, Zhao and Austin (2005) noted that the spin of the toroidal circulation downshear flank was enhanced relative to the upshear flank. While these influences of shear on thermal behavior are notable, it is unclear how they may impact convective development. Further investigation is needed to develop a cohesive view of the "practical" influences of shear on convective development, such as how shear generally influences entrainment/detrainment and the ascent rate of thermals. This question provides the primary motivation for our study.

The influence of shear on thermals does not just pertain to convective initiation. Though "slab-like" lifting may occur in the lowest few kilometers of the leading edge of squall lines (e.g., James et al. 2005), highresolution simulations $[O(100)$-m horizontal and vertical spacing] almost universally show that lifted slab-like layers (e.g., Kingsmill and Houze 1999) break down into thermals in the middle- to upper troposphere (e.g., Bryan et al. 2003, 2007; Bryan and Morrison 2012; Peters 2016; Lebo and Morrison 2015). The presence of vertical wind shear in most squall-line environments often leads to convection slanting rearward of the system's leading edge (e.g., Rotunno et al. 1988). Previous authors have argued that this rearward tilt should make vertical accelerations and vertical velocities weaker than in the case of upright convection, all else being equal, because of larger downward-directed buoyant perturbation pressure acceleration for slanted convection (Parker 2010; Peters 2016). However, these authors considered idealized "plumelike" updrafts. It is unclear whether the conclusions from these studies hold for squall lines composed of a series of quasi-spherical thermals rising along a slanted path, as indicated by high-resolution modeling, rather than continuous, slanted plumelike updrafts. This knowledge gap further 
motivates investigating the dynamical response of thermals to a vertically sheared environment.

To address the aforementioned questions, we devised a simple numerical modeling experiment to compare the attributes of simulated moist thermals in sheared and unsheared environments. The following specific hypotheses are addressed:

1) Dilution of thermals' thermodynamic properties from entrainment and detrainment reduces thermals' buoyancies, which makes them ascend at slower rates.

2) Dilution of thermals' vertical momentum from momentum entrainment and detrainment directly reduces their ascent rates.

3) Vertical wind shear enhances the downward-directed dynamic pressure gradient force (e.g., Morrison and Peters 2018, hereafter MP) that opposes thermals' buoyancy-driven ascent.

4) Sheared thermals rise in a slantwise path, which reduces their effective buoyancy and their ascent rates, akin to the effect described by Parker (2010) and Peters (2016).

Note that while both hypotheses 1 and 2 relate to entrainment and detrainment, they represent two distinct effects of entrainment and detrainment on thermals' properties and are often discussed separately in the scientific literature. We therefore address these effects individually.

The organization of this paper is as follows. Section 2 describes the numerical modeling setup, an algorithm that was used to track thermals, and the dynamical analysis framework used to evaluate the thermals' momentum budgets. The results of these simulations and analyses are described in section 3 . Section 4 provides a summary, discussion, and conclusions.

\section{Experiment design}

To evaluate our hypotheses, we first ran a series of idealized cloud model simulations where deep convection was initialized in unsheared and sheared environments. We then tracked thermals within these simulations and evaluated their vertical momentum budgets to determine how vertical accelerations differed between the sheared and unsheared runs. The numerical modeling setup is described in section $2 \mathrm{a}$, the thermal tracking algorithm in section $2 \mathrm{~b}$, and the framework for assessing thermals' momentum budgets is introduced in section $2 \mathrm{c}$.

\section{a. Simulations}

Our simulations use Cloud Model 1 (CM1; Bryan and Fritsch 2002), version 18. CM1 is nonhydrostatic and designed for idealized numerical modeling experiments. The dynamical core uses a compressible equation set with acoustic time splitting (e.g., Klemp and Wilhelmson 1978). For each simulation, 264 grid points were used with a grid spacing of $100 \mathrm{~m}$ in the $x, y$, and $z$ directions. Lower and upper boundaries were free slip and lateral boundaries were periodic. Microphysical process were parameterized using the double-moment scheme described in Morrison et al. (2009), with graupel as the prognostic rimed-ice hydrometeor species. Simulations were initialized with the background state set to the well-known analytic sounding of WK82 (hereafter the WK82 profile), with a boundary layer water vapor mixing ratio of $14 \mathrm{~g} \mathrm{~kg}^{-1}$ (Fig. 1a). This sounding was used because its convective available potential energy (CAPE) of $\approx 2000 \mathrm{~J} \mathrm{~kg}^{-1}$ reflects the midlatitude continental convective environments that typically experience moderate to strong vertical wind shear, although we note that it is much moister than typical midlatitude continental environments at mid- to upper levels. Convection was initiated using the method of Hannah (2017) by applying a 1-K Gaussian-shaped warm and moist bubble centered horizontally within the initial conditions at a height of $500 \mathrm{~m}$. A horizontally constant relative humidity was assumed everywhere within the initial conditions, which determined the moisture perturbation within the warm bubble. Random temperature perturbations with a maximum amplitude of $1 \mathrm{~K}$ were included in the initial conditions below $2 \mathrm{~km}$, which allowed the simulations to quickly develop realistic turbulence. To generate a sufficiently large sample size, our simulation set includes four different sets of initial random noise and three different initial bubble radii $(1,1.5$, and $2 \mathrm{~km})$ meaning that there were 12 unique simulations for each wind profile (the different wind profile configurations are discussed below). All simulations were run for $20 \mathrm{~min}$. A summary of the modeling configuration is provided in Table 1.

The base-state wind profile was constant at $0 \mathrm{~m} \mathrm{~s}^{-1}$ in the NOSHR (for "no shear") runs to represent an unsheared environment (Fig. 1b). In the SHR (for "shear") simulations, the base state $u$ wind was $-5 \mathrm{~m} \mathrm{~s}^{-1}$ at the surface, increased linearly to $25 \mathrm{~m} \mathrm{~s}^{-1}$ at $9 \mathrm{~km}\left(\partial u_{0} / \partial z=\right.$ $3.3 \times 10^{-3} \mathrm{~s}^{-1}$ ), and remained constant at $25 \mathrm{~m} \mathrm{~s}^{-1}$ above $9 \mathrm{~km}$ (Fig. 1b). Additional runs with other shear magnitudes were analyzed, but these runs are not described here as they do not change our conclusions. We therefore concentrate on comparing attributes of the NOSHR runs to the SHR runs with $\partial u_{0} / \partial z=3.3 \times 10^{-3} \mathrm{~s}^{-1}$.

\section{b. Thermal tracking algorithm}

We tracked individual moist thermals using a method similar to that of DS. First, model data were written at an 

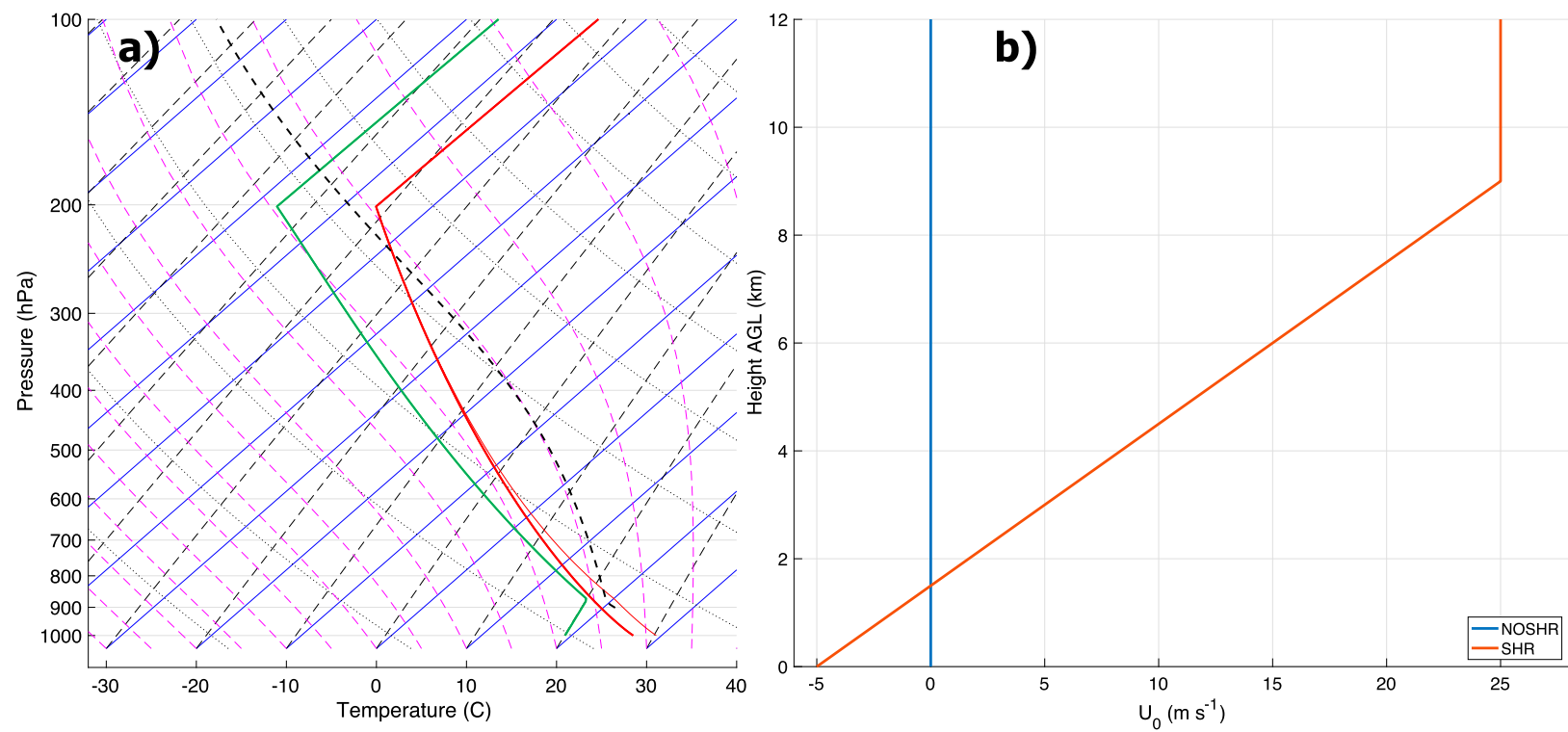

FIG. 1. (a) Skew-T- $\log P$ diagram of the WK82 sounding used for the simulations in this study, with temperature $\left({ }^{\circ} \mathrm{C}\right)$, virtual temperature $\left({ }^{\circ} \mathrm{C}\right)$, dewpoint temperature $\left({ }^{\circ} \mathrm{C}\right)$, and the temperature of a lifted parcel with the average properties of the lowest $1 \mathrm{~km}\left({ }^{\circ} \mathrm{C}\right)$ shown as thick red, thin red, green, and black dashed lines, respectively. (b) Profiles of initial $u$ wind $\left(u_{0} ; \mathrm{m} \mathrm{s}^{-1}\right)$ for the NOSHR (blue) and SHR (red) runs.

output frequency of $15 \mathrm{~s}$. Within each model output time, we found local maxima in vertical velocity that exceeded $3 \mathrm{~m} \mathrm{~s}^{-1}$, representing the largest vertical velocity within a 500-m-wide cube centered at the local maxima value. These local maxima points were cataloged as "thermal center candidates" (TCCs). Each TCC with center location $x_{1}, y_{1}, z_{1}$ was then matched with the closest TCC at the next time step with center $x_{2}, y_{2}, z_{2}$ if that TCC was within the range $x_{1}-1.5 w_{1} \Delta t<x_{2}<x_{1}+1.5 w_{1} \Delta t$, $y_{1}-1.5 w_{1} \Delta t<y_{2}<y_{1}+1.5 w_{1} \Delta t$, and $z_{2}+\Delta x<z_{2}<$ $z_{1}+1.5 w_{1} \Delta t$, where $w_{1}$ is the vertical velocity at the TCC at the initial time step, $\Delta x=100 \mathrm{~m}$, and $\Delta t=15 \mathrm{~s}$. The identical process was then performed in reverse. If both the forward-in-time and backward-in-time methods identified the same matching TCCs, then these TTCs were considered part of the same thermal trajectory. Thermal trajectories with less than three points in time were discarded. The thermal ascent rate was then determined via a temporally centered finite difference approximation using the thermal's center locations. At each time step, the thermal's boundary was defined as a sphere centered at the thermal center whose volume-averaged $w$ was equal to the thermal's ascent rate at that time. Resulting thermal trajectories were then further evaluated with the momentum budget analysis described below.

\section{c. Momentum budget}

To arrive at an equation for the vertical acceleration of moist thermals, we begin with the vertical momentum equation for an anelastic atmosphere:

$$
\frac{\partial w}{\partial t}=-\frac{1}{\rho_{0}} \nabla \cdot\left(\rho_{0} \mathbf{U} w\right)-\frac{1}{\rho_{0}} \frac{\partial p_{d}}{\partial z}-\frac{1}{\rho_{0}} \frac{\partial p_{b}}{\partial z}+B+\mathrm{SGS},
$$

where $B \equiv-g \rho^{\prime} / \rho_{0}-g \sum q_{i}$ is buoyancy and $q_{i}$ is the $i$ th hydrometeor species, SGS represents subgrid-scale turbulence and numerical diffusion,

TABLE 1. Summary of the CM1 V18 configuration.

\begin{tabular}{lll}
\hline \multicolumn{1}{c}{ Attribute } & Value/setting & Notes \\
\hline Horizontal grid spacing & $100 \mathrm{~m}$ & \\
Vertical grid spacing & $100 \mathrm{~m}$ & \\
Vertical coordinate & Height $(\mathrm{m})$ & \\
Number of $x$ and $y$ points & $264 \times 264$ & \\
Vertical points & 264 & \\
Top/bottom boundary & Free-slip & (2005) \\
$\quad$ conditions & Periodic \\
Lateral boundary & \\
$\quad$ conditions & Morrison \\
Microphysics & Sixth order \\
& Smagorinski \\
Diffusion & Yes \\
Subgrid turbulence & Yes \\
Rayleigh dampening & $75-0.04$ \\
Dissipative heating & \\
Second- and sixth-order & - \\
$\quad$ difference coefficient & - \\
Longwave radiation & - \\
Shortwave radiation & - \\
Surface layer & - \\
Boundary layer physics & - \\
Cumulus parameterization & - \\
\hline
\end{tabular}




$$
\begin{aligned}
& \nabla^{2} p_{b}=\frac{\partial\left(\rho_{0} B\right)}{\partial z}, \\
& \nabla^{2} p_{d}=-\nabla \cdot\left(\rho_{0} \mathbf{U} \cdot \nabla\right) \mathbf{U},
\end{aligned}
$$

and all other terms retain their traditional meanings. The SGS term was generally small because of the momentum budget restrictions placed on analyzed thermals (discussed later in this section), so this term is ignored hereafter. Equations (2) and (3) were solved by discretizing the Laplacian on a centered second-order finite difference grid, performing a two-dimensional fast Fourier transform in the horizontal direction, solving the resulting tridiagonal difference equation in the vertical, and then inverting the Fourier transform (this is the exact method used in CM1 to solve the pressure equation when the model is run in anelastic mode).
Next, we introduce the matrix $\boldsymbol{\sigma}$, which is set to 1 within a thermal and 0 elsewhere. Multiplying Eq. (1) by $\boldsymbol{\sigma}$, rearranging terms using calculus identities, and then volume integrating over the model domain gives

$$
\begin{aligned}
\frac{\partial\langle w\rangle_{c}}{\partial t}= & \left\langle w \frac{\partial \sigma}{\partial t}\right\rangle-\left\langle\frac{1}{\rho_{0}} \nabla \cdot\left(\rho_{0} \mathbf{U} w\right)\right\rangle_{c} \\
& -\left\langle\frac{1}{\rho_{0}} \frac{\partial p_{d}}{\partial z}\right\rangle_{c}-\left\langle\frac{1}{\rho_{0}} \frac{\partial p_{b}}{\partial z}\right\rangle_{c}+\langle B\rangle_{c}
\end{aligned}
$$

where for an arbitrary quantity $\phi$, we define $\langle\phi\rangle \equiv$ $\iiint \phi d V_{d}$ where $V_{d}$ is the volume of the model domain. We also note that $\langle\boldsymbol{\sigma} \phi\rangle=\langle\phi\rangle_{c}$, where $\langle\phi\rangle_{c}$ is the volume integral over the thermal. Dividing Eq. (4) by $\langle\boldsymbol{\sigma}\rangle=V$ (where $V$ is the thermal volume) and rearranging terms gives

$$
\frac{\partial \bar{w}}{\partial t}=\underbrace{\frac{\tilde{w}-\bar{w}}{V_{c}} \frac{\partial V}{\partial t}+\frac{1}{V} \oiint_{\Omega} w \mathbf{U} \cdot \nabla A d \Omega}_{\text {Term A: Entrainment/detrainment of } w} \underbrace{-\frac{\overline{1} \frac{\partial p_{d}}{\rho_{0}} \frac{\partial z}{\rho_{0}}}{-\frac{1}{\rho_{0}} \frac{\partial p_{b}}{\partial z}}}_{\text {Term B: DPA }}+\underbrace{\overline{\bar{B}}}_{\text {Term C: BPA }},
$$

where $\bar{\phi} \equiv\langle\phi\rangle / V, \Omega$ is the surface of the thermal, and $\tilde{w} \equiv \oiint_{\Omega} w d \Omega / \oiint_{\Omega} d \Omega$ represents $w$ averaged over the thermal boundary. Because $\sigma$ is defined so that $\bar{w}$ is equal to the thermal's ascent rate, Eq. (5) describes the physical processes that regulate a thermal's vertical acceleration:

- Term A: $w$ tendency due to momentum entrainment/ detrainment. If hypothesis 2 were supported, this term would contribute larger downward acceleration in sheared runs than in unsheared runs.

- Term B: Dynamic pressure acceleration (DPA). This typically acts in opposition to $B$ (e.g., MP). If hypothesis 3 were supported, we would expect larger downward acceleration from this term in the sheared runs than in the unsheared runs.

- Term C: Buoyancy pressure acceleration (BPA). This also acts in opposition to $B$ (e.g., Morrison 2016a,b; Peters 2016). If hypothesis 4 were supported, we would expect larger downward acceleration from this term in sheared runs than in unsheared runs.

- Term D: Buoyancy accelerations. If hypothesis 1 were supported, we would expect smaller thermal buoyancies at a given height in the sheared runs than in the unsheared runs indicating comparatively larger entrainment-driven dilution of buoyancy in the sheared runs.

For a thermal to be considered in subsequent analysis, we required that the vertical displacement of the thermal over its lifespan predicted by the integrated momentum budget was no greater than $20 \%$ different than the actual vertical displacement of the thermal.

\section{Analysis of simulations}

Simulated cloudy updrafts were notably turbulent, owing to the inclusion of random noise within the initial conditions (Figs. 2a,c). To quantify turbulence, we computed the Fourier energy spectrum (e.g., Bryan et al. 2003; Lebo and Morrison 2015) using horizontal two-dimensional discrete Fourier transforms (DFT) of $(1 / 2) w^{2}$ on each model level between 3 and $7 \mathrm{~km}$. The DFT is written as

$$
\psi(k, j)=\frac{\Delta x}{2} \sum_{n=0}^{N-1} \sum_{m=0}^{M-1} w_{n, m}^{2} e^{-(2 \pi i / N) k n-(2 \pi i / M) j m},
$$

where $N=M=264$ and $k$ and $j$ are spatial frequencies in the $x$ and $y$ directions. The Fourier energy spectrum was then computed from the DFT coefficients $\psi(k, j)$ using the following formula:

$$
E(r)=\frac{1}{\beta} \sum \psi(k, j) \quad \text { for } \quad r-\Delta r \leq \sqrt{k^{2}+j^{2}}<r+\Delta r,
$$

where $r$ is a radial frequency, $\beta$ is the number of grid points in Fourier space that satisfy $r-\Delta r \leq$ $\sqrt{k^{2}+j^{2}}<r+\Delta r, \Delta x$ is the grid spacing, and $\Delta r$ was set to $1 / 2$. Here, $E(r)$ quantifies the amount of energy within 

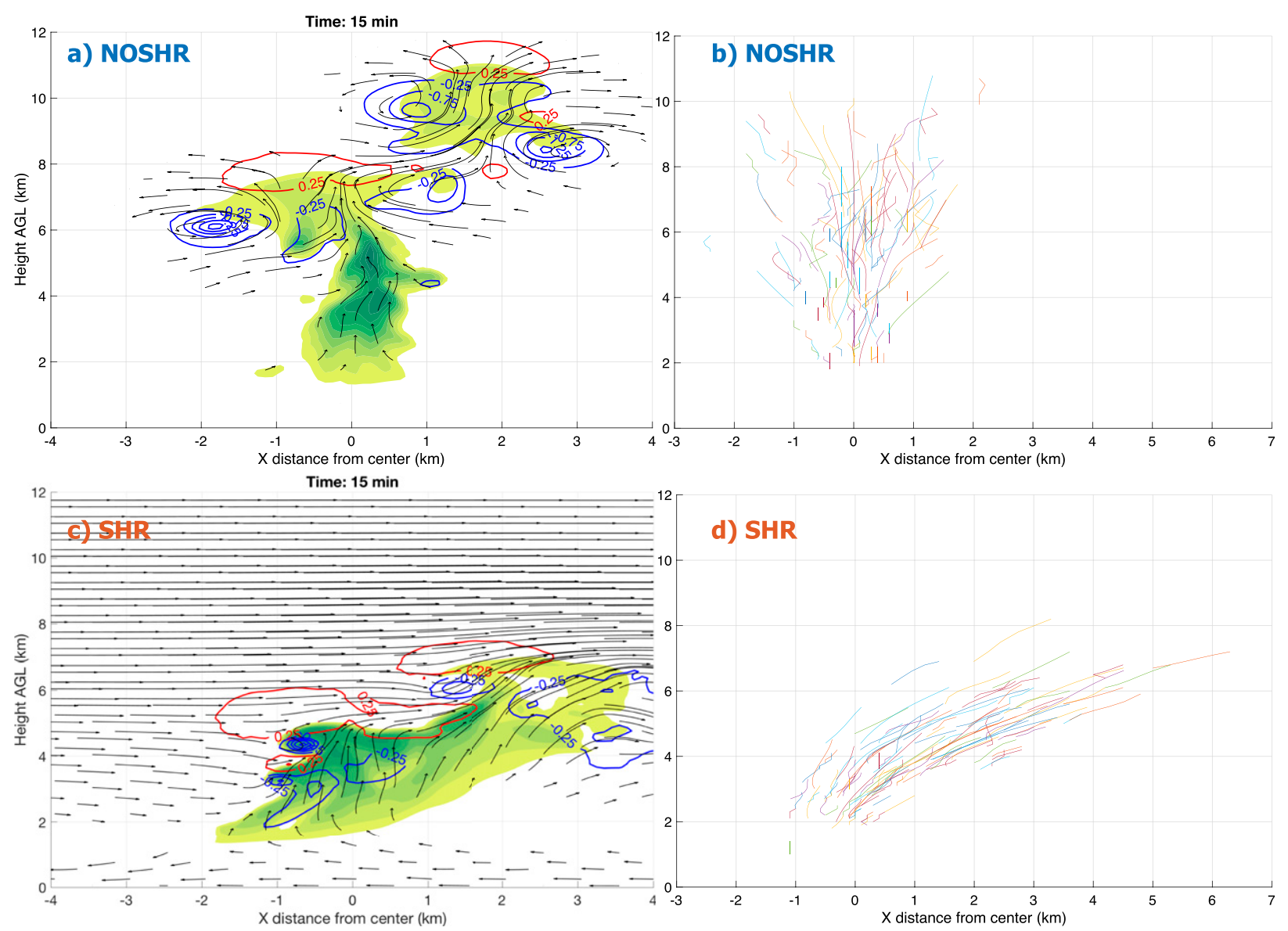

FIG. 2. (a),(c) Cross sections along the center of the domain in the $y$ direction valid after 15 min of model integration, showing cloud and ice water mixing ratios (shading; $\mathrm{kg} \mathrm{kg}^{-1}$ ), pressure perturbations (negative is blue, positive is red; intervals of $0.25 \mathrm{hPa}$, with the 0 - $\mathrm{hPa}$ contour omitted), and streamlines (black arrows) from selected simulations with 2-km initial bubbles. (b), (d) Paths in the $x-z$ plane of tracked thermals from all simulations (lines). (a),(b) NOSHR runs and (c),(d) SHR runs.

the domain that corresponds to phenomena with an effective wavelength of $1 / r$. According to Kolmogorov's scaling, $E(r)$ should have a slope proportional to $r^{-5 / 3}$ for scales within the inertial subrange that are larger than the lower effective resolution bound [e.g., 6-7 $\Delta x$ in Bryan et al. (2003)]. This is indeed true for a large portion of the frequency space in the SHR and NOSHR runs. The $E(r)$ slopes are shallower than $r^{-5 / 3}$ for wavelengths larger than about $1 \mathrm{~km}$, and peak near $8 \mathrm{~km}$. These scales correspond to relatively large, energy containing eddies on the scale of the convective updrafts themselves. Overall, the closeness of the $E(r)$ spectral slopes to $r^{-5 / 3}$ at scales smaller than $\sim 1 \mathrm{~km}$ but larger than the effective resolution instills confidence that the simulations are depicting realistic turbulence (Fig. 3a).

Well-defined thermal-like circulations were subjectively apparent in instantaneous snapshots of model output. A total of 382 and 372 thermals were tracked and passed the momentum budget criterion in the NOSHR and SHR runs, respectively. In the NOSHR runs, thermals moved up to $2 \mathrm{~km}$ radially from the domain center because of turbulent processes (Fig. 2b). Unsurprisingly, thermals in the SHR runs were transported much larger distances downstream of the domain center by increasing "westerly" (left to right) wind speeds in the middle to upper troposphere (Fig. 2d). The size distributions of tracked thermals peaked at $500-\mathrm{m}$ radii for all runs (Fig. 3a), which is similar to previous studies using large domain large-eddy simulations (e.g., DS). However, the largest percentage of vertical mass flux was accomplished by thermals closer to $1 \mathrm{~km}$ in radius (thin red and blue lines in Fig. 3a), which is a similar to the overall radius of the clouds in our simulations (e.g., Figs. 2a,c). Thermal start and end heights varied considerably, which is consistent with previous studies (e.g., DS), and the average thermal lifetimes were 63 and $72 \mathrm{~s}$ in the NOSHR and SHR runs, respectively. Note that the end 


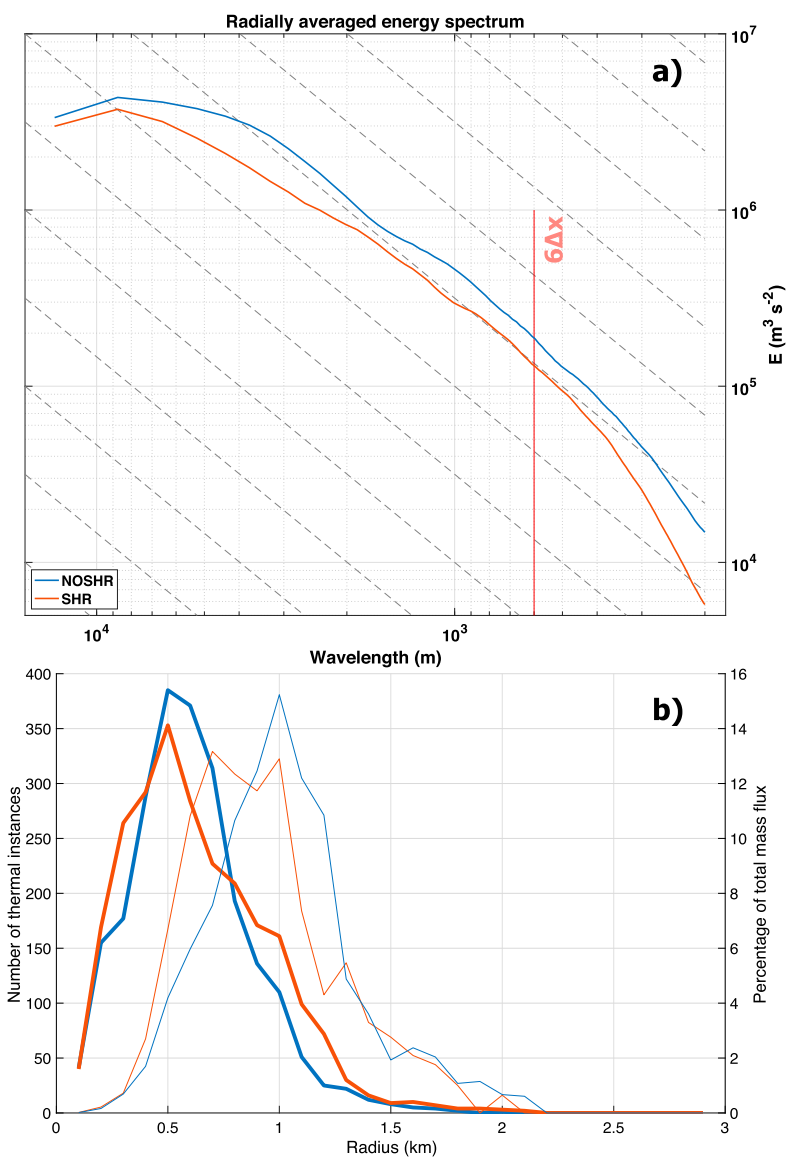

FIG. 3. (a) Plots of $E(r)$ from Eq. (7) for the 2-km bubble initializations. Curves with a slope of $-5 / 3$ are shown as gray dashed lines. (b) Distributions of the number of thermal instances (thick lines) as a function of thermal radius, and the percentage of total thermal mass flux accomplished by thermals within a given radius bin (thin lines). Bin widths are $250 \mathrm{~m}$. A "thermal instance" in (b) represents a snapshot from 15 -s model output of a given tracked thermal. In both panels the NOSHR run is blue and SHR run is red.

points of tracked thermals in the SHR runs were considerably lower than those of the NOSHR runs, and reasons for this difference will be discussed in detail later in this section. Vertical mass flux (MF)-weighted composites of thermal wind, pressure, and buoyancy show a classical spherical vortex-like flow structure and associated perturbation pressure minima appearing as two lobes in vertical cross-section plots (Figs. 4a,b). This ring vortex-like structure of moist thermals was also found in previous large-eddy simulations of deep convection (Sherwood et al. 2013; Romps and Charn 2015; DS).

In subsequent analyses of height distributions of quantities, thermals were binned every $500 \mathrm{~m}$, starting at $250 \mathrm{~m}$ in height. MF-weighted averages of quantities were then computed for each bin. Because of the MFweighted averaging, our analysis intrinsically focuses on

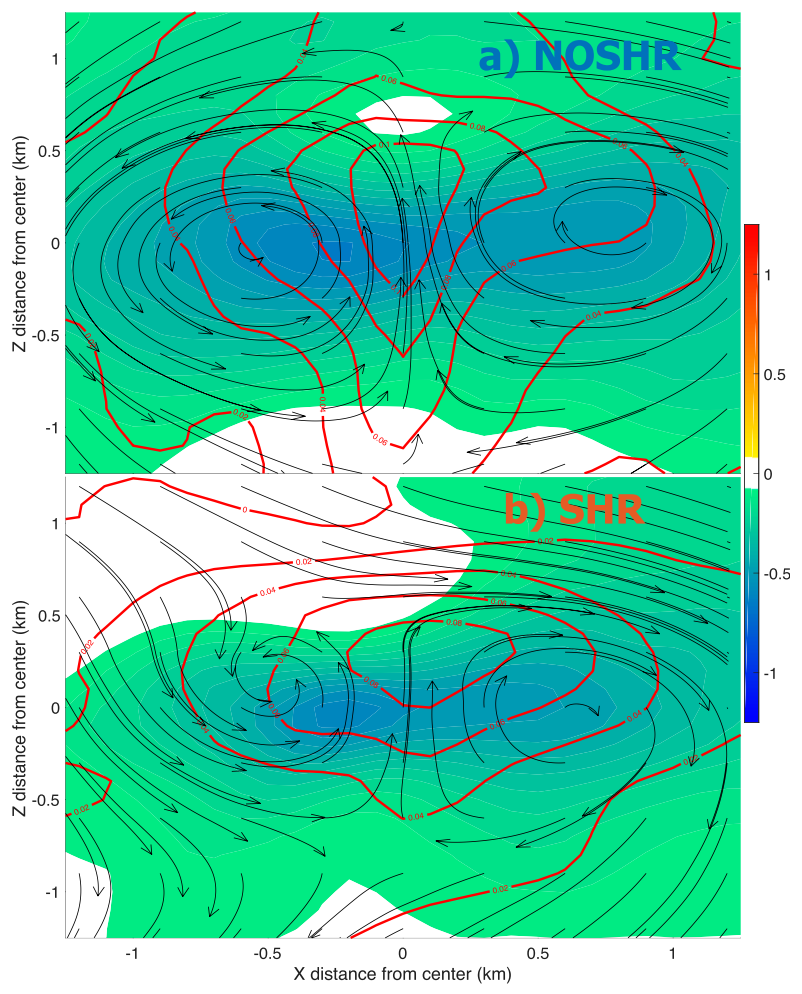

FIG. 4. Vertical mass flux-weighted composites of dynamic pressure perturbations (shading; $\mathrm{hPa}$ ), buoyancy (red contours at intervals of $0.02 \mathrm{~m} \mathrm{~s}^{-2}$ ), and $u$ and $w$ streamlines relative to the thermal's $u$ and $w$ motion components. (a) The NOSHR runs and (b) the SHR runs.

the thermals that are the most important contributions to vertical mass redistribution accomplished by the cloud. The results were unchanged when thermals with $R<500 \mathrm{~m}$ were omitted from our analysis, suggesting that the results described hereafter were minimally dependent on thermal size. Statistical significance of differences between the SHR and NOSHR runs was assessed via a bootstrap method. In this method, the distributions of quantities in given height bins were resampled 1000 times in both the SHR and NOSHR runs based on a uniform random distribution with the potential for repeating indices, and each time the differences of the averages or sums were recomputed between the SHR and the NOSHR runs. If the 5th and 95th percentiles of these differences retained the same sign, then a difference was considered statistically significant.

\section{a. Momentum budget analysis}

The vertical distributions of total thermal MF show much larger MF in the NOSHR runs above $5 \mathrm{~km}$ than in the SHR runs (Figs. 5a,d). Comparable peak MF magnitudes in the SHR runs occurred 3-4 km lower than those in the NOSHR runs, showing that the 

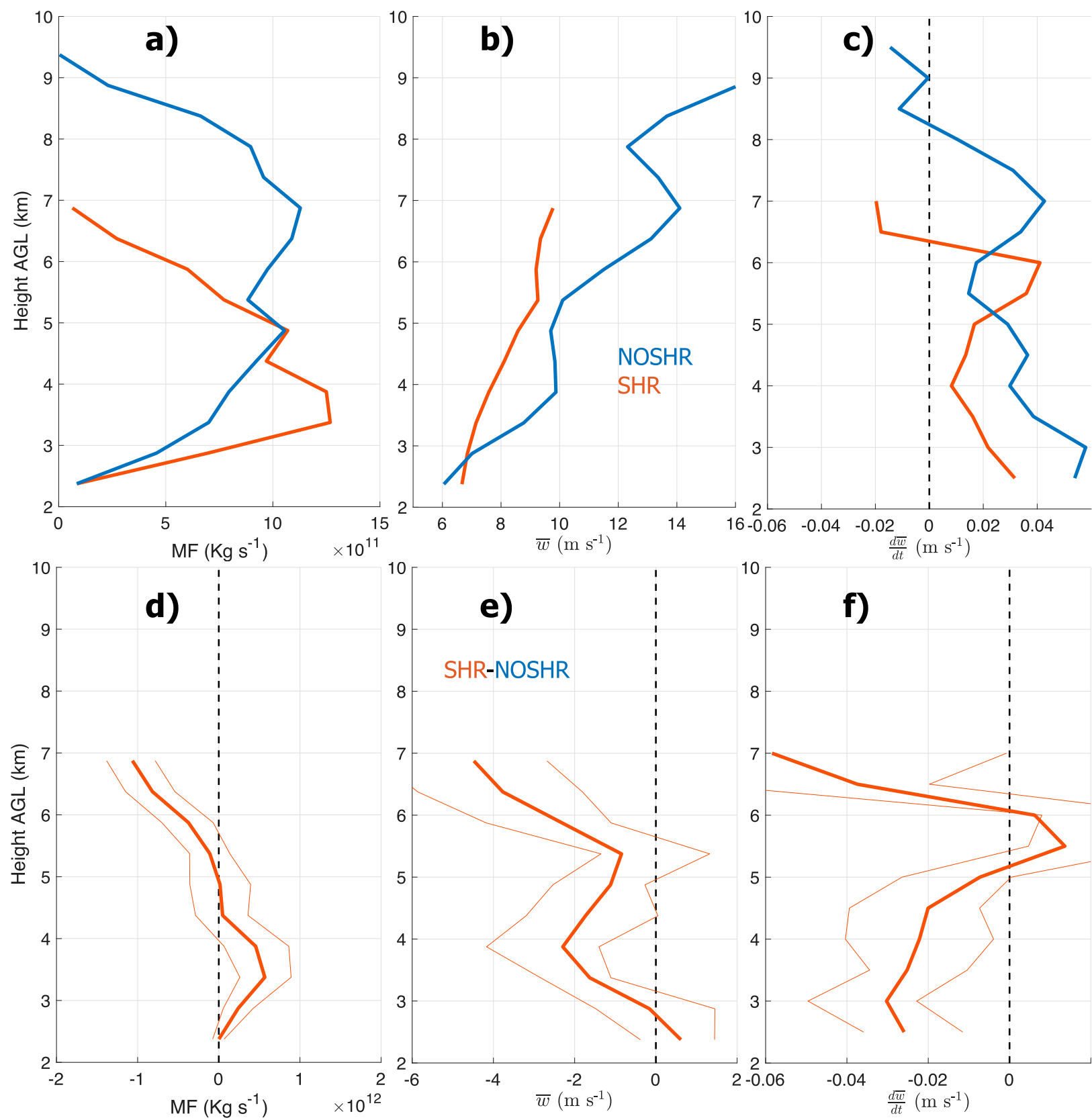

FIG. 5. (a)-(c) Vertical profiles of total thermal vertical mass flux $\left(\mathrm{kg} \mathrm{s}^{-1}\right)$, average $w\left(\mathrm{~m} \mathrm{~s}^{-1}\right)$, and average $d W / d t$ ( $\left.\mathrm{m} \mathrm{s}^{-2}\right)$, respectively. (d)-(f) Differences between the SHR (thick blue lines) and the NOSHR runs, and the 5th- and 95th-percentile differences determined via the bootstrapping procedure described in the text (thin lines) of the quantities in (a)-(c). For a difference to be statistically significant, both thin lines must lie on the same side of the zero line.

presence of vertical wind shear in the SHR runs inhibited the rate of cloud deepening compared to the NOSHR runs. Indeed, the vertical distributions of $\bar{w}$ (Figs. 5b,e) and $d \bar{w} / d t$ (Figs. 5c,f) were statistically significantly smaller in the SHR runs than in the NOSHR runs, suggesting that vertical wind shear reduced the depth of cloud growth by reducing thermals' upward accelerations.
Buoyancy contributed large upward accelerations in the lower troposphere, and decreased in magnitude with height (Figs. 6a,e). This is consistent with previous studies of thermals' momentum budgets (e.g., DS). BPA was negative in all simulations (Figs. 6b,f), which is consistent with past studies that have shown BPA should approximately scale as $-(1 / 3) B$ (Tarshish et al. 2018). Interestingly, MP showed generally smaller downward 

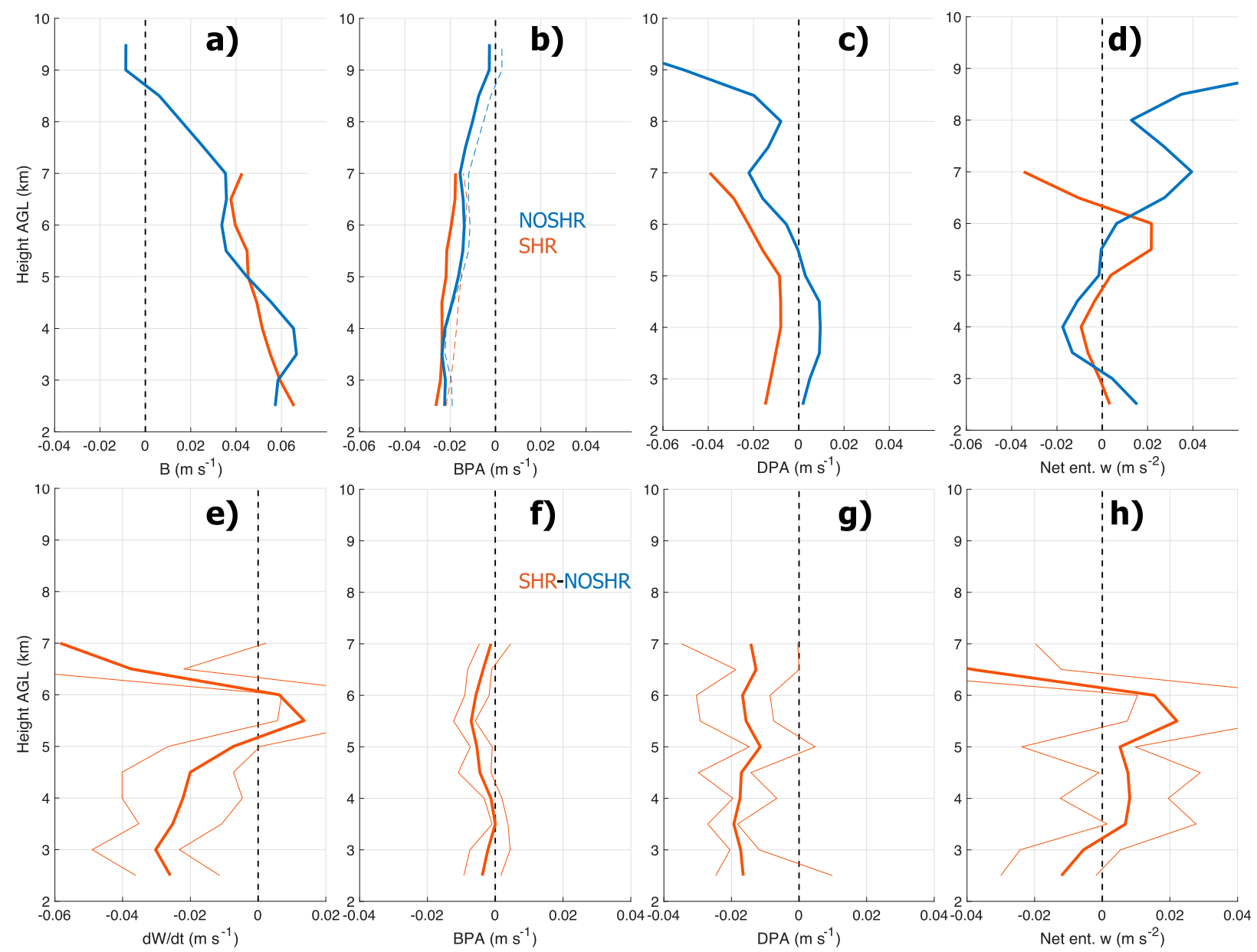

FIG. 6. As in Fig. 5, but for averages of (a),(e) $B\left(\mathrm{~m} \mathrm{~s}^{-2}\right)$, (b),(f) BPA $\left(\mathrm{m} \mathrm{s}^{-2}\right)$, and (c),(g) DPA ( $\left.\mathrm{m} \mathrm{s}^{-2}\right)$, and (d), (h) net $w$ tendency from momentum entrainment and detrainment.

BPA magnitudes in simulations of axisymmetric thermals than those found here. It is possible that the presence of large positively buoyant "stems" in the wake of thermals influenced their analysis of BPA, and such stems may have been disrupted by turbulence in the simulations analyzed here. DPA was also generally downward, except for below $5 \mathrm{~km}$ in the NOSHR simulations (Figs. 6c,g), which also contrasts with the findings of MP who showed DPA to be universally downward for positively buoyant axisymmetric thermals. This discrepancy may again result from the azimuthal homogeneity in the simulations of MP. DPA magnitudes generally became more negative with height as thermals' ascent rates increased, which is consistent with the results of MP and DS. Accelerations from momentum entrainment/detrainment were smaller in magnitude in the SHR runs than the NOSHR runs below $5 \mathrm{~km}$, and briefly larger in the SHR runs than the NOSHR runs between 5 and $6 \mathrm{~km}$ and near $7 \mathrm{~km}$ (Figs. 6d,h).
The presence of shear generally impacted all terms of the $w$ budget. Thermals in the SHR runs were generally less buoyant than those in the NOSHR simulations in the 3-5-km layer, though these differences vanished above $5 \mathrm{~km}$ (Figs. 6a,e). There were also statistically significant differences in BPA between the SHR and NOSHR runs; however, these differences were small compared to the differences in other acceleration terms (Figs. 6b,f). BPA in the NOSHR runs corresponded well to $-(1 / 3) B$, whereas BPA in the SHR runs was slightly more negative than $-(1 / 3) B$, indicating some enhancement of BPA in the SHR runs relative to the NOSHR runs. DPA was uniformly more negative in the SHR runs than in the NOSHR runs (Figs. 6c,g). Accelerations from entrainment and detrainment processes were more negative in the SHR runs than the NOSHR runs below $3 \mathrm{~km}$, but became more positive above $3 \mathrm{~km}$ (Figs. 6d,h). However, these entrainment/ detrainment differences were only statistically significant at 2.5 and $3.5 \mathrm{~km}$ and above $6.6 \mathrm{~km}$. 

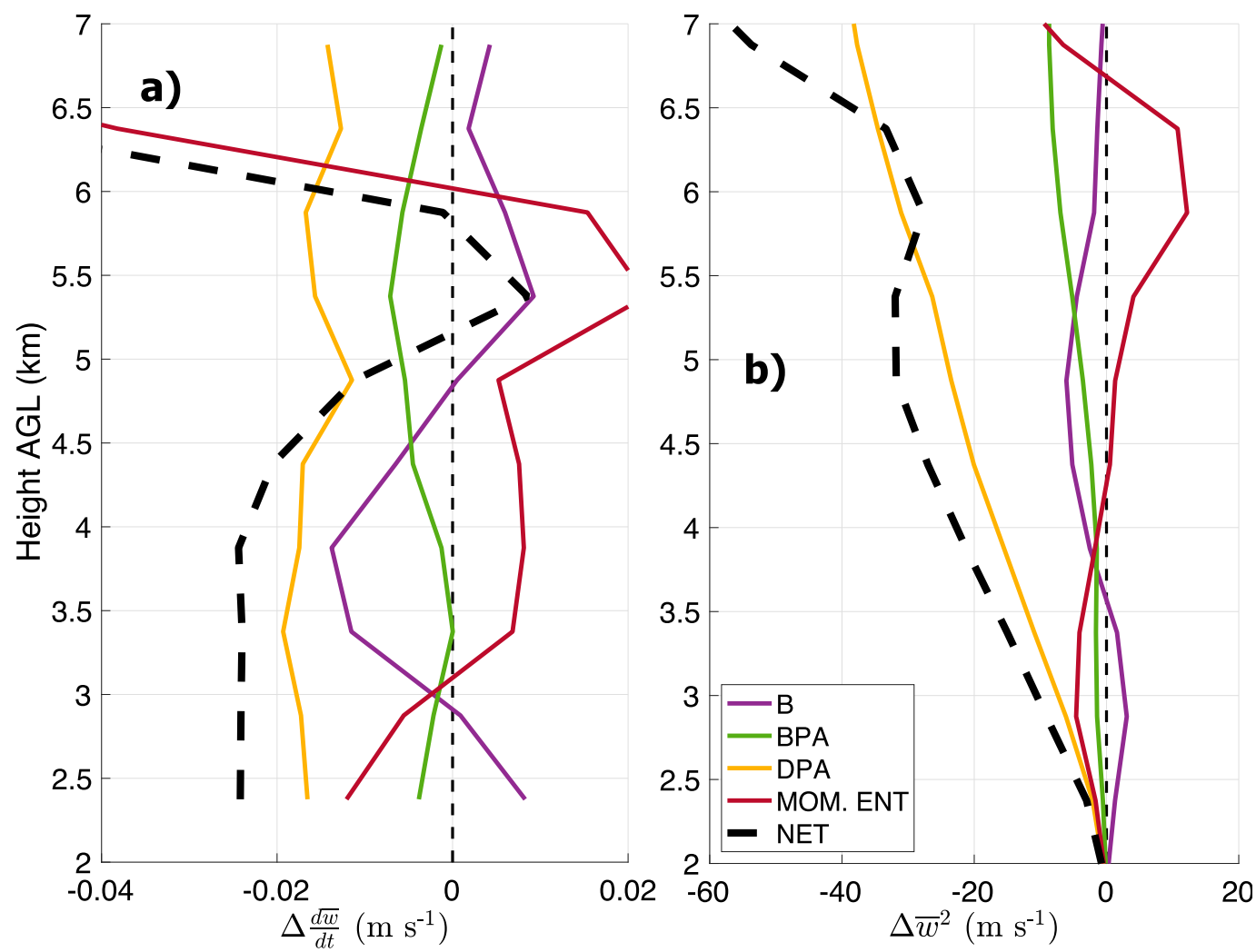

FIG. 7. (a) Side-by-side comparisons of the differences between the SHR and NOSHR runs in $B$ (purple lines; $\mathrm{m} \mathrm{s}^{-2}$ ), BPA (green lines; $\mathrm{m} \mathrm{s}^{-2}$ ), DPA (orange lines; $\mathrm{m} \mathrm{s}^{-2}$ ), total entrainment/detrainment (dark red lines; $\mathrm{m} \mathrm{s}^{-2}$ ), and the sum of all terms (thick black dashed lines; $\mathrm{m} \mathrm{s}^{-2}$ ) with the NOSHR runs. (b) The vertical integral of the quantities in (a).

In general, the largest differences in the momentum budgets between the SHR and NOSHR runs were greater downward-directed DPA in SHR than NOSHR, and to a lesser extent smaller buoyancy in SHR (Fig. 7a). These differences were partially compensated by momentum entrainment/detrainment, which produced somewhat greater downward-directed acceleration in the NOSHR runs than SHR. Furthermore, the large negative difference in net upward acceleration between the SHR and NOSHR runs corresponded well with the differences in DPA between these runs (Fig. 7a). If we assume that the vertical distributions of the acceleration terms are steady with time so that $d \bar{w} / d t=\bar{w} d \bar{w} / d z=$ $(1 / 2) d \bar{w}^{2} / d z$, we may better understand the influence that these acceleration terms have on the thermals' ascent rates by vertically integrating Eq. (5), yielding a contribution to $\bar{w}^{2}$ by each acceleration term. Because of the consistently more negative DPA in the SHR runs than in the NOSHR runs, DPA was the predominant contributor to the smaller $\bar{w}^{2}$ in the SHR runs than in the NOSHR runs (Fig. 7b). The analysis in this section therefore supports hypothesis 3, indicating that shear enhances downward DPA, which reduces the rate of cloud growth in sheared environments compared to unsheared environments.

\section{b. Dynamics responsible for enhanced downward $D P A$ in the sheared runs}

In this section, we provide evidence that differences in accelerations from aerodynamic lift between the sheared and unsheared simulations are responsible for the enhancement in downward DPA acting upon the sheared thermals. Potential flow theory dictates that a dynamic pressure gradient force arises when flow passes along one side of an object at a faster rate than along the opposite side. When oriented in the vertical direction, this pressure gradient force is referred to as aerodynamic "lift." In the case of flow that encounters a spinning object, the flow will be slowed down on one side of the object relative to the other because of differences in friction, resulting in the lift force associated with the well-known "Magnus effect." This is the principle that makes curveballs deviate from straight trajectories in baseball. Previous authors have shown that forces analogous to aerodynamic lift have a measurable influence on laboratory ring vortices 
(Chang and Vakili 1995) and observed cloud thermals (Damiani and Vali 2007).

The dynamic pressure force acting upon the toroidal circulation of a thermal can be illustrated by considering steady-state two-dimensional Boussinesq flow that moves from left to right, and that encounters a vortex with negative horizontal vorticity. The equation for the thermal-relative horizontal flow in steady state is

$$
\frac{\partial\left(\frac{1}{2} u^{2}+\alpha_{0} p^{\prime}\right)}{\partial x}+w \frac{\partial u}{\partial z}=0,
$$

where $\alpha_{0} \equiv 1 / \rho_{0}$. Boundary conditions are $u=u_{0}$ and $p^{\prime}=0$ at $x=-\infty$ and $u=u_{0}+u^{\prime}$ at the $x$ location aligned with the center of the vortex $x=c$ on the upstream side of the thermal. Horizontally integrating from $x=-\infty$ to $x=c$ along lines that intersect the top $\left(z=z_{t}\right)$ and bottom $\left(z=z_{b}\right)$ of the vortex gives

$$
\begin{gathered}
\left(u_{0} u^{\prime}\right)_{z=z_{t}}+\frac{1}{2} u_{z=z_{t}}^{\prime 2}+\alpha_{0} p_{x=c, z=z_{t}}^{\prime}=0, \\
\left(u_{0} u^{\prime}\right)_{z=z_{b}}+\frac{1}{2} u_{z=z_{b}}^{\prime 2}+\alpha_{0} p_{x=c, z=z_{b}}^{\prime}=0,
\end{gathered}
$$

where we have assumed that $w$ is small within the bounds of integration. We define $\Delta u$ as the horizontal wind difference between the bottom and the top of the vortex, such that $u_{z=z_{t}}^{\prime}=-(1 / 2) \Delta u$ and $u_{z=z_{t}}^{\prime}=(1 / 2) \Delta u$. Likewise, $\Delta p \equiv p_{x=c, z=z_{t}}^{\prime}-p_{x=c, z=z_{b}}^{\prime}$. Subtracting Eq. (10) from Eq. (9), substituting in our wind and pressure definitions, and solving for $\Delta p$ gives

$$
\Delta p=\rho_{0} u_{\mathrm{CF}} \Delta u,
$$

where the cross flow is defined as $2 u_{\mathrm{CF}} \equiv u_{0, z=z_{t}}+u_{0, z=z_{b}}$. Because $\Delta u>0$ on the upstream side of the thermal's toroidal circulation, a positive $u_{\mathrm{CF}}$ (i.e., a net cross flow from left to right) will result in a larger pressure on top of the upstream side of the toroidal circulation than on the bottom. For there to be nonzero cross flow, the thermal's motion in the $x$ direction must be different than the $u$ wind speed averaged through the thermal's depth. An estimate for the pressure gradient acceleration that results from the pressure difference is obtained by rearranging and then dividing by the diameter of the toroidal circulation $D_{\text {tor }} \approx R$ (where $R$ is the radius of the thermal):

$$
\mathrm{DPA}_{\mathrm{LIFT}} \approx-\frac{\Delta p}{R \rho_{0}}=-\frac{u_{\mathrm{CF}} \Delta u}{R},
$$

where DPA $A_{\text {LIFT }}$ is the DPA imparted by the lift acceleration, which is negative (downward). Interestingly, thermals in both runs experienced generally increasing cross-flow magnitudes with height. The presence of a net cross flow in the NOSHR runs may seem counter intuitive given the absence of background flow, but this simply results from thermals in the NOSHR runs drifting from their initial starting horizontal positions and moving through the stationary background flow (i.e., Figs. 2a,b). As a consequence of the net cross flow in both sets of runs, both the NOSHR and SHR thermals experienced lift forces (note the tilted toroidal circulations apparent in both Figs. 2a and 2c). Cross-flow magnitudes in the SHR runs at a given height, on the other hand, were nearly double the cross-flow magnitudes of the NOSHR runs, suggesting that the SHR thermals experienced comparatively stronger lift forces.

For an isolated thermal, $\Delta u$ will be positive on the upstream side of the toroid and negative on the downstream side of the toroid, implying equal and opposite forces imparted on the thermal and hence no impact on the net vertical acceleration. However, composites from the SHR runs show that the cross flow below the thermal was "blocked" by convectively modified air below the thermal and did not reach the bottom of the downstream part of the toroidal circulation (Fig. 9d). This meant that a downward lift force was present on the upstream side of the toroid, but no lift was present on the downstream side of the toroid.

The flow structure for the SHR runs implies that $u_{\mathrm{CF}} \neq 0$ and $\mathrm{DPA}_{\mathrm{LIFT}}<0$ on the upstream side of the thermal, but $u_{\mathrm{CF}} \approx 0$ and $\mathrm{DPA}_{\mathrm{LIFT}} \approx 0$ on the downstream side of the thermal. This pattern of lift forces should therefore impart a net downward force on the thermals in the SHR runs. This footprint of the lift force in the SHR runs manifests as vertical and horizontal perturbation pressure asymmetries in the composite thermal. If the cross-flow magnitude were identical on both the upstream and downstream sides of the toroidal circulation, a bias toward low (high) pressure should be present on the lower (upper) parts of the upstream side of the thermal, and a bias toward high (low) pressure should be present on the lower (upper) parts of the downstream side of the thermal (these biases are relative to a thermal experiencing zero net cross flow). If the magnitude of the lift force was larger on the upstream side of the thermal, the vertical pressure asymmetry should be much more pronounced on the upstream side of the thermal than on the downstream side. Indeed we find this to be the case for the SHR composite thermal structure (Figs. 9e,f), with relatively low (high) dynamic perturbation pressure on the lower-left (upper left) side of the thermal, and a much less pronounced vertical asymmetry of dynamic perturbation pressure on the right (downwind) side of the thermal. These patterns of 

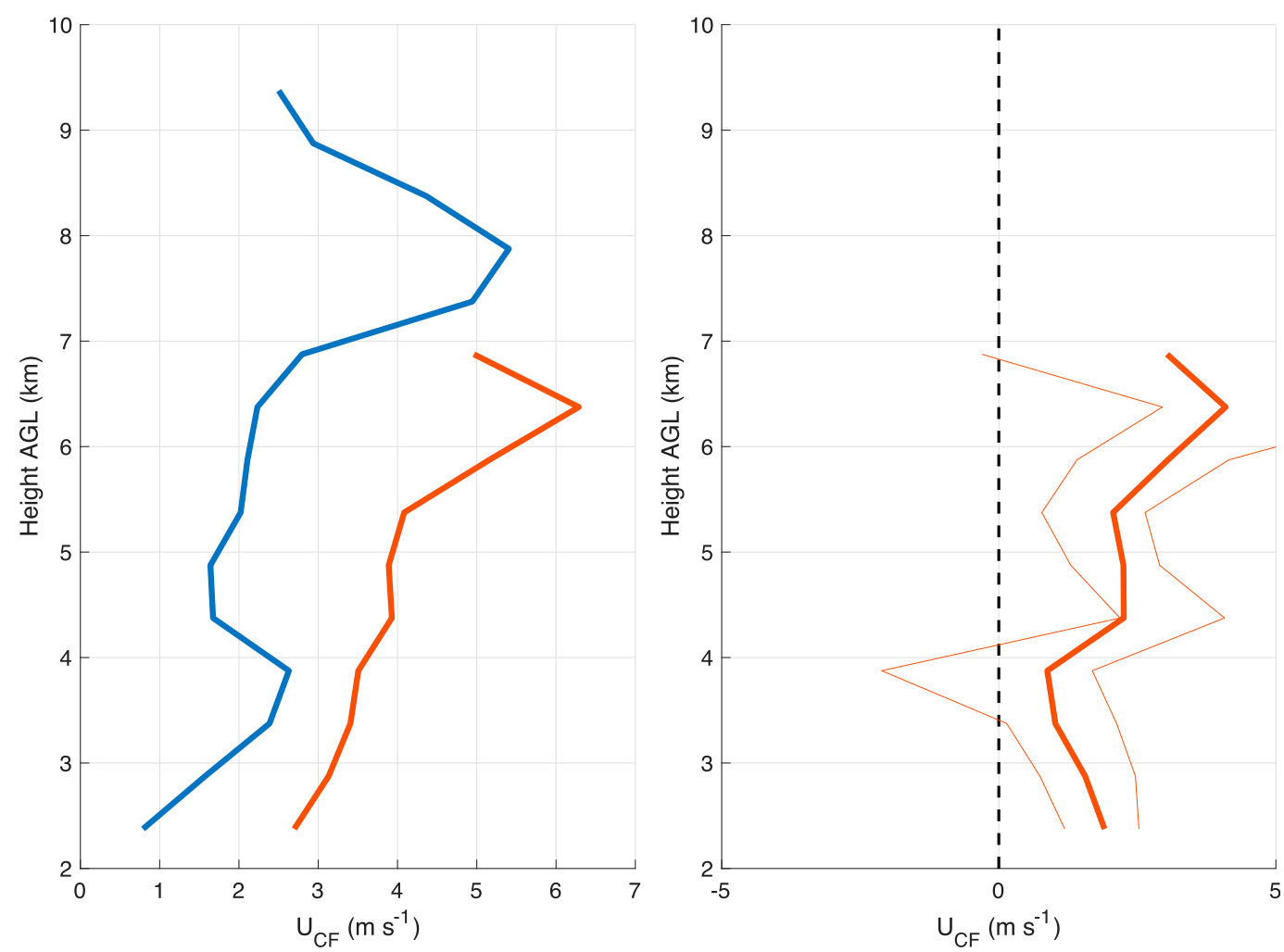

FIG. 8. (a) As in Fig. 6, but for the magnitude of the net cross flow defined as $u_{\mathrm{CF}}(z) \equiv\left|u_{0}(z)-U(z)\right|$, where $U(z)$ is a thermal's $u$ velocity at a given height $z$ and $u_{0}$ is the wind associated with the background shear at $z$.

cross flow and vertical pressure asymmetry are well contrasted with the almost vertically antisymmetric $u$ (Fig. 9a) wind and symmetric dynamic perturbation pressure fields (Figs. 9b,c) fields for the NOSHR thermals. As noted above, there are likely weak net lift forces acting upon the NOSHR thermals because of relative cross flow. However, with no environmental shear there is no preferred orientation of the cross flow and hence almost no horizontal asymmetry of dynamic pressure.

The magnitude of the lift force acting upon thermals is quantified via estimates of the individual quantities on the right-hand side of Eq. (12). We estimate $u_{\mathrm{CF}}=$ 4-6 $\mathrm{m} \mathrm{s}^{-1}$ from Fig. 8a, $\Delta u=4-6 \mathrm{~m} \mathrm{~s}^{-1}$ from Fig. 9d, and $\rho_{0}=0.8-0.6 \mathrm{~kg} \mathrm{~m}^{-3}$. From Eq. (11), the pressure asymmetry $\Delta p$ associated with lift forces ranges from 0.13 to $0.29 \mathrm{hPa}$. This estimate is consistent with the $\Delta p_{d}^{\prime} \sim 0.17 \mathrm{hPa}$ from the composite SHR runs in Fig. 9 . Assuming that $\mathrm{DPA}_{\mathrm{LIFT} \text {,downstream }}=0, \mathrm{DPA}_{\mathrm{LIFT} \text {,upstream }}$ $<0$, and the net downward lift force on the thermal is $(1 / 2) \mathrm{DPA}_{\mathrm{LIFT} \text {,upstream }}$, we get $\mathrm{DPA}_{\mathrm{LIFT}} \approx-0.008$ to $-0.021 \mathrm{~m} \mathrm{~s}^{-1}$ (this assumes $R=0.5-1 \mathrm{~km}$ ). This is consistent with the differences in DPA between the sheared and unsheared runs in Fig. 6c, although our simple estimate of the net lift force would be somewhat smaller if $3 \mathrm{D}$ effects were considered given that the component of the cross flow aligned with the toroidal circulation scales approximately with the cosine of the angle between the two. Overall, these results suggest that lift forces were the primary reason for the larger downward DPA in the sheared runs than in the unsheared runs.

Another possible factor that was influenced by shear is wave drag. Thermals generate gravity waves as they rise through stratified environments, which exert a back force on the thermal that can act in opposition to thermals' upward motion. This force should manifest in both the distributions of DPA (because of the kinematic circulations associated with gravity waves) and BPA (because of the buoyancy structure associated with gravity waves). The wave drag coefficient $c_{d}$ is nonlinearly dependent on the Froude number [Romps and Oktem 2015; DS; $F_{r} \equiv|\mathbf{V}| /(N R)$, where $N \equiv \sqrt{(g / \theta)(\partial \theta / \partial z)}$ and $\left.|\mathbf{V}| \equiv \sqrt{u_{\mathrm{CF}}^{2}+\bar{w}^{2}}\right]$. Because the background thermodynamic environment was the same in the SHR and NOSHR simulations, potential differences in $F_{r}$ must have resulted from differences in the cross-flow magnitude and ascent rates between the two simulations. Given that cross-flow magnitudes were $2-3 \mathrm{~m} \mathrm{~s}^{-1}$ larger (Fig. $8 \mathrm{~b}$ ), but ascent rates were 

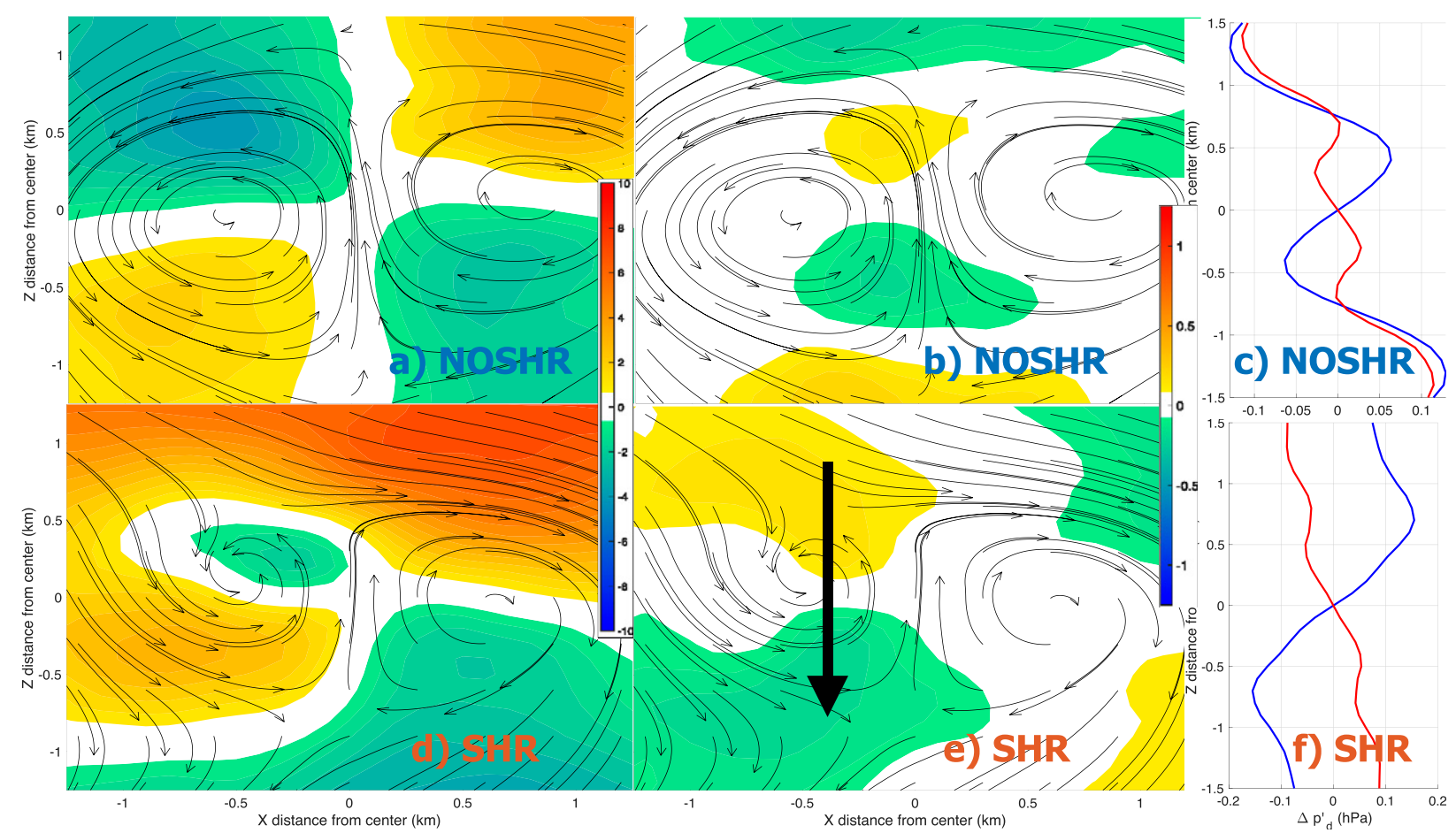

FIG. 9. (a),(d) Composites of the $u$ wind component (shading; $\mathrm{m} \mathrm{s}^{-1}$ ), and $u$ and $w$ streamlines relative to the thermal's $u$ - and $w$-motion components. (b),(e) Composites of the vertical asymmetry in the dynamic perturbation pressure field $\Delta p_{d}^{\prime}$ (shading; hPa), defined as the dynamic perturbation pressure at a given distance above (below) the thermal center line minus that at the same distance below (above) the centerline $\left[\Delta p_{d}^{\prime} \equiv p_{d}(x, y, z)-p_{d}\left(x, y, 2 z_{\text {cent }}-z\right)\right.$, where $z_{\text {cent }}$ is the height of the composite center]. All other fields are as in (a) and (d), but with buoyancy contoured in red. (c),(f) Plot of $\Delta p_{d}^{\prime}$ averaged in the $x$ direction between -1.5 and $0 \mathrm{~km}$ (blue line) and averaged between 0 and $1.5 \mathrm{~km}$ in the $x$ direction (red line). (a)-(c) The NOSHR runs and (d)-(f) the SHR runs. The thick black arrow in (e) annotates the downward-oriented lift force.

2-3 $\mathrm{m} \mathrm{s}^{-1}$ smaller (Fig. 5e) in the SHR runs than in the NOSHR runs, it is likely that $|\mathbf{V}|, F_{r}$, and wave drag were not much different between the two simulations. This assertion is supported by the fact that, if there were appreciable differences in wave drag between the simulations, we would expect large differences in BPA between the runs-yet these differences were small relative to differences in other quantities (Fig. 6).

\section{Summary, conclusions, and discussion}

Previous research and anecdotal observations have hypothesized that convection develops at a slower rate in vertically sheared environments than in unsheared environments. We evaluate this hypothesis by comparing the ascent rates and momentum budgets of moist thermals-which themselves are an essential element to growing convection-in the sheared and unsheared simulations. Our conclusions are as follows:

1) Thermals reach altitudes $3-4 \mathrm{~km}$ higher in unsheared runs than sheared runs, and the vertical mass flux is biased toward higher altitudes in unsheared runs.
This demonstrates that shear does indeed impede the rate of deepening of moist convection.

2) The lower termination heights of sheared thermals results from sheared thermals having smaller vertical accelerations and ascent rates.

3) Sheared thermals have stronger downward dynamic pressure acceleration than unsheared thermals, which explains the largest fraction of their smaller accelerations and ascent rates compared to unsheared thermals.

4) Forces that were analogous to aerodynamic lift associated with horizontal cross flow (relative to the thermal trajectory) are the likely reason why sheared thermals have stronger downward dynamic pressure acceleration than unsheared thermals.

Our results contrast with the notion that the primary way shear inhibits convective development is by enhancing entrainment-driven dilution, at least on the scale of individual cloud thermals. In our simulations, the influence of entrainment-related processes on thermals' ascent rates was small relative to dynamic pressure acceleration. It is possible that entrainment may play 
a larger role in diluting buoyancy in a drier middle troposphere than the environments considered here, and future work should investigate this potential sensitivity. Previous authors have also assumed that slanted convection should be weaker than upright convection because of the enhanced downward buoyant pressure acceleration of the former (e.g., Parker 2010; Peters 2016). However, we find here that despite the large degree to which convection in the sheared runs was slanted relative to the unsheared runs, the primary difference in downward-directed accelerations between these two runs was in dynamic pressure acceleration rather than in buoyant pressure acceleration. A potential explanation for this discrepancy is that previous authors assumed that slanted updrafts have a plumelike structure, whereas the convection in our simulations occurs as a semidiscrete series of rising quasi-spherical thermals in both the sheared and unsheared runs. The buoyant pressure acceleration associated with buoyancy anomalies for the geometry of a slanted plume are quite different from those associated with nearly spherical buoyancy anomalies. Future work should apply the thermal tracking procedures used here to squall-line simulations to explore this idea further. Finally, our results are potentially applicable to improving cumulus parameterizations. If future cumulus parameterizations are constructed with thermals (rather than steady-state plumes) as the basic unit of convection, the thermals' vertical momentum could be formulated to include the effects of environmental wind shear and cross flow.

Acknowledgments. This work was performed under the auspices of the U.S. Department of Energy by Lawrence Livermore National Laboratory under Contract DE-AC52-07NA27344. J. Peters's efforts were partially supported by the National Science Foundation Grant AGS-1841674. The National Center for Atmospheric Research is sponsored by the National Science Foundation.

\section{REFERENCES}

Brooks, H. E., C. A. Doswell III, and R. Davies-Jones, 1993: Environmental helicity and the maintenance and evolution of low-level mesocyclones. The Tornado: Its Structure, Dynamics, Prediction, and Hazards, Geophys. Monogr., Vol. 79, Amer. Geophys. Union, 97-104.

Bryan, G. H., and J. M. Fritsch, 2002: A benchmark simulation for moist nonhydrostatic numerical models. Mon. Wea. Rev., 130, 2917-2928, https://doi.org/10.1175/1520-0493(2002)130<2917: ABSFMN $>2.0 . \mathrm{CO} ; 2$.

, and H. Morrison, 2012: Sensitivity of a simulated squall line to horizontal resolution and parameterization of microphysics. Mon. Wea. Rev., 140, 202-225, https://doi.org/10.1175/MWRD-11-00046.1.
_ and R. Rotunno, 2014: The optimal state for gravity currents in shear. J. Atmos. Sci., 71, 448-468, https://doi.org/10.1175/ JAS-D-13-0156.1.

—- J. C. Wyngaard, and J. M. Fritsch, 2003: Resolution requirements for the simulation of deep moist updrafts. Mon. Wea. Rev., 131, 2394-2416, https://doi.org/10.1175/ 1520-0493(2003)131<2394:RRFTSO>2.0.CO;2.

— J. C. Knievel, and M. D. Parker, 2006: A multimodel assessment of RKW theory's relevance to squall-line characteristics. Mon. Wea. Rev., 64, 1249-1266, https://doi.org/ 10.1175/MWR3226.1

— R. Rotunno, and J. M. Fritsch, 2007: Roll circulations in the convective region of a simulated squall line. J. Atmos. Sci., 64, 1249-1266, https://doi.org/10.1175/JAS3899.1.

Bunkers, M. J., B. A. Klimowski, R. L. Thompson, and M. L. Weisman, 2000: Predicting supercell motion using a new hodograph technique. Wea. Forecasting, 15, 61-79, https://doi.org/10.1175/15200434(2000)015<0061:PSMUAN >2.0.CO;2.

Chang, Y. K., and A. D. Vakili, 1995: Dynamics of vortex rings in crossflow. Phys. Fluids, 7, 1583-1597, https://doi.org/10.1063/ 1.868545 .

Coniglio, M. C., H. E. Brooks, S. J. Weiss, and S. F. Corfidi, 2007: Forecasting the maintenance of quasi-linear mesoscale convective systems. Wea. Forecasting, 22, 556-570, https://doi.org/ 10.1175/WAF1006.1.

Damiani, R., and G. Vali, 2007: Evidence for tilted toroidal circulations in cumulus. J. Atmos. Sci., 64, 2045-2060, https:// doi.org/10.1175/JAS3941.1.

_- and Coauthors, 2008: The Cumulus, Photogrammetric, In Situ, and Doppler Observations experiment of 2006. Bull. Amer. Meteor. Soc., 89, 57-73, https://doi.org/10.1175/ BAMS-89-1-57.

Davies-Jones, R., 1984: Streamwise vorticity: The origin of updraft rotation in supercell storms. J. Atmos. Sci., 41, 2991-3006, https:// doi.org/10.1175/1520-0469(1984)041<2991:SVTOOU>2.0.CO;2.

— 2002: Linear and nonlinear propagation of supercell storms. J. Atmos. Sci., 59, 3178-3205, https://doi.org/10.1175/ 1520-0469(2003)059<3178:LANPOS > 2.0.CO;2.

Fric, T. F., and A. Roshko, 1994: Vortical structure in the wake of a transverse jet. J. Fluid Mech., 279, 1-47, https://doi.org/ 10.1017/S0022112094003800.

Hane, C. E., 1973: The squall line thunderstorm: Numerical experimentation. J. Atmos. Sci., 30, 1672-1690, https://doi.org/ 10.1175/1520-0469(1973)030<1672:TSLTNE $>2.0$. CO;2.

Hannah, W. M., 2017: Entrainment vs. dilution in tropical deep convection. J. Atmos. Sci., 74, 3725-3747, https://doi.org/ 10.1175/JAS-D-16-0169.1.

Hernandez-Deckers, D., and S. C. Sherwood, 2016: A numerical investigation of cumulus thermals. J. Atmos. Sci., 73, 41174136, https://doi.org/10.1175/JAS-D-15-0385.1.

James, R. P., J. M. Fritsch, and P. M. Markowski, 2005: Environmental distinctions between cellular and slabular convection. Mon. Wea. Rev., 133, 2669-2691, https://doi.org/10.1175/MWR3002.1.

Kingsmill, D. E., and R. A. Houze Jr., 1999: Thermodynamic characteristics of air flowing into and out of precipitating convection over the west Pacific warm pool. Quart. J. Roy. Meteor. Soc., 125, 1209-1229, https://doi.org/10.1002/qj.1999.49712555606.

Klemp, J. B., and R. B. Wilhelmson, 1978: Simulations of rightand left-moving storms produced through storm splitting. J. Atmos. Sci., 35, 1097-1110, https://doi.org/10.1175/15200469(1978)035<1097:SORALM > 2.0.CO;2.

Lebo, Z. J., and H. Morrison, 2015: Effects of horizontal and vertical grid spacing on mixing in simulated squall lines and 
implications for convective strength and structure. Mon. Wea. Rev., 143, 4355-4375, https://doi.org/10.1175/MWR-D-15-0154.1.

Markowski, P., and Y. Richardson, 2010: Mesoscale Meteorology in Midlatitudes. Wiley-Blackwell, $430 \mathrm{pp}$.

Moncrieff, M. W., and C. Liu, 1999: Convection initiation by density currents: Role of convergence, shear, and dynamical organization. Mon. Wea. Rev., 127, 2455-2464, https://doi.org/ 10.1175/1520-0493(1999)127<2455:CIBDCR >2.0.CO;2.

Morrison, H., 2016a: Impacts of updraft size and dimensionality on the perturbation pressure and vertical velocity in cumulus convection. Part I: Simple, generalized analytic solutions. J. Atmos. Sci., 73, 1441-1454, https://doi.org/10.1175/JAS-D-15-0040.1.

_ 2016b: Impacts of updraft size and dimensionality on the perturbation pressure and vertical velocity in cumulus convection. Part II: Comparison of theoretical and numerical solutions. J. Atmos. Sci., 73, 1455-1480, https://doi.org/10.1175/JAS-D-15-0041.1.

— , and J. M. Peters, 2018: Theoretical expressions for the ascent rate of moist convective thermals. J. Atmos. Sci., 75, 16991719, https://doi.org/10.1175/JAS-D-17-0295.1.

— J. A. Curry, and V. I. Khvorostyanov, 2005: A new doublemoment microphysics parameterization for application in cloud and climate models. Part I: Description. J. Atmos. Sci., 62, 1665-1677, https://doi.org/10.1175/JAS3446.1.

_ , G. Thompson, and V. Tatarskii, 2009: Impact of cloud microphysics on the development of trailing stratiform precipitation in a simulated squall line: Comparison of one- and two-moment schemes. Mon. Wea. Rev., 137, 991-1007, https:// doi.org/10.1175/2008MWR2556.1.

Moser, D. H., and S. Lasher-Trapp, 2017: The influence of successive thermals on entrainment and dilution in a simulated cumulus congestus. J. Atmos. Sci., 74, 375-392, https://doi.org/ 10.1175/JAS-D-16-0144.1.

Pan, D.-M., and D. A. Randall, 1998: A cumulus parameterization with a prognostic closure. Quart. J. Roy. Meteor. Soc., 124, 949-981, https://doi.org/10.1002/qj.49712454714.

Parker, M. D., 2010: Relationship between system slope and updraft intensity in squall lines. Mon. Wea. Rev., 138, 3572-3578, https://doi.org/10.1175/2010MWR3441.1.

Peters, J. M., 2016: The impact of effective buoyancy and dynamic pressure forcing on vertical velocities within two-dimensional updrafts. J. Atmos. Sci., 73, 4531-4551, https://doi.org/10.1175/ JAS-D-16-0016.1.

Rasmussen, E. N., and J. M. Straka, 1998: Variations in supercell morphology. Part I: Observations of the role of upper-level storm-relative flow. Mon. Wea. Rev., 126, 2406-2421, https:// doi.org/10.1175/1520-0493(1998)126<2406:VISMPI >2.0.CO;2.

Romps, D. M., and A. B. Charn, 2015: Sticky thermals: Evidence for a dominant balance between buoyancy and drag in cloud updrafts. J. Atmos. Sci., 72, 2890-2901, https://doi.org/10.1175/ JAS-D-15-0042.1.

— , and R. Oktem, 2015: Stereo photogrammetry reveals substantial drag on cloud thermals. Geophys. Res. Lett., 42, 50515057, https://doi.org/10.1002/2015GL064009.

Rotunno, R., and J. B. Klemp, 1982: The influence of the shearinduced pressure gradient on thunderstorm motion. Mon. Wea. Rev., 110, 136-151, https://doi.org/10.1175/1520-0493(1982) $110<0136$ :TIOTSI $>2.0 . \mathrm{CO} ; 2$.

,-- , and M. L. Weisman, 1988: A theory for strong, longlived squall lines. J. Atmos. Sci., 45, 463-485, https://doi.org/ 10.1175/1520-0469(1988)045<0463:ATFSLL > 2.0.CO;2.

Sau, R., and K. Mahesh, 2008: Dynamics and mixing of vortex rings in crossflow. J. Fluid Mech., 604, 389-409, https://doi.org/ 10.1017/S0022112008001328.
Sherwood, S. C., D. Hernandez-Deckers, and M. Colin, 2013: Slippery thermals and the cumulus entrainment paradox. J. Atmos. Sci., 70, 2426-2442, https://doi.org/10.1175/JASD-12-0220.1.

Smith, B. T., R. L. Thompson, J. S. Grams, C. Broyles, and H. E. Brooks, 2012: Convective modes for significant severe thunderstorms in the contiguous United States. Part I: Storm classification and climatology. Wea. Forecasting, 27, 11141135, https://doi.org/10.1175/WAF-D-11-00115.1.

Takeda, T., 1966: Effects of the prevailing wind and vertical shear on the convective cloud accompanied with heavy rainfall. J. Meteor. Soc. Japan, 44, 129-144, https://doi.org/10.2151/ jmsj1965.44.2_129.

Tarshish, N., N. Jeevanjee, and D. Lecoanet, 2018: Buoyant motion of a turbulent thermal. J. Atmos. Sci., 75, 3233-3244, https:// doi.org/10.1175/JAS-D-17-0371.1.

Thompson, R. L., R. Edwards, J. A. Hart, K. L. Elmore, and P. Markowski, 2003: Close proximity soundings within supercell environments obtained from the Rapid Update Cycle. Wea. Forecasting, 18, 1243-1261, https://doi.org/10.1175/ 1520-0434(2003)018<1243:CPSWSE > 2.0.CO;2.

C. M. Mead, and R. Edwards, 2007: Effective storm-relative helicity and bulk shear in supercell thunderstorm environments. Wea. Forecasting, 22, 102-115, https://doi.org/10.1175/WAF969.1.

- B. T. Smith, J. S. Grams, A. R. Dean, and C. Broyles, 2012: Convective modes for significant severe thunderstorms in the contiguous United States. Part II: Supercell and QLCS tornado environments. Wea. Forecasting, 27, 1136-1154, https:// doi.org/10.1175/WAF-D-11-00116.1.

Thorpe, A. J., M. J. Miller, and M. W. Moncrieff, 1982: Twodimensional convection in non-constant shear: A model for mid-latitude squall lines. Quart. J. Roy. Meteor. Soc., 108, 739-762, https://doi.org/10.1002/qj.49710845802.

Trapp, R. J., G. R. Marion, and S. W. Nesbitt, 2017: The regulation of tornado intensity by updraft width. J. Atmos. Sci., 74, 41994211, https://doi.org/10.1175/JAS-D-16-0331.1.

Warren, R. A., H. Richter, H. A. Ramsay, S. T. Siems, and M. J. Manton, 2017: Impact of variations in upper-level shear on simulated supercells. Mon. Wea. Rev., 145, 2659-2681, https:// doi.org/10.1175/MWR-D-16-0412.1.

Weisman, M. L., 1992: The role of convectively generated rearinflow jets in the evolution of long-lived mesoconvective systems. J. Atmos. Sci., 49, 1826-1847, https://doi.org/10.1175/ 1520-0469(1992)049<1826:TROCGR > 2.0.CO;2.

_ 1993: The genesis of severe, long-lived bow echoes. J. Atmos. Sci., 50, 645-670, https://doi.org/10.1175/1520-0469(1993) $050<0645$ :TGOSLL $>2.0$.CO;2.

—_, and J. B. Klemp, 1982: The dependence of numerically simulated convective storms on vertical wind shear and buoyancy. Mon. Wea. Rev., 110, 504-520, https://doi.org/ 10.1175/1520-0493(1982)110<0504:TDONSC >2.0.CO;2.

— and - 1984: The structure and classification of numerically simulated convective storms in directionally varying wind shears. Mon. Wea. Rev., 112, 2479-2498, https://doi.org/ 10.1175/1520-0493(1984)112<2479:TSACON $>2.0$.CO;2.

, and R. Rotunno, 2000: The use of vertical wind shear versus helicity in interpreting supercell dynamics. J. Atmos. Sci., 57, 1452-1472, https://doi.org/10.1175/1520-0469(2000)057<1452: TUOVWS $>2.0 . \mathrm{CO} ; 2$.

Zhao, M., and P. H. Austin, 2005: Life cycle of numerically simulated shallow cumulus clouds. Part II: Mixing dynamics. J. Atmos. Sci., 62, 1291-1310, https://doi.org/10.1175/ JAS3415.1. 